\title{
Elementos que inciden en el proceso de adaptación académica según la percepción de un grupo de estudiantes de primer ingreso de la UNED de la asignatura Métodos de Estudio a Distancia e Investigación
}

José Pablo Meza-Pérez *

https://orcid.org/0000-0001-6752-048X

Recibido: 21 de julio de 2020 • Aceptado: 16 de octubre de 2020

\section{Resumen}

En este artículo se estudia el fenómeno de la adaptación a la UNED de los estudiantes de primer ingreso que cursaron la asignatura Métodos de Estudio a Distancia e Investigación (código 00055) en el segundo cuatrimestre del 2019 desde el análisis de las siguientes categorías: a) Categorización del estudiante en relación e integración de los principios de la educación a distancia de autorregulación, autoconocimiento y motivación académica, b) Categorización de la percepción de los estudiantes en el proceso de enseñanza aprendizaje realizado en la asignatura 0055, c) Identificación de las instancias a las cuales debió acudir este cuatrimestre para acompañar su proceso de enseñanza aprendizaje, y d) Acogida en el sistema de educación a distancia. Se concluye sobre la necesidad de mejorar el proceso de mediación de los aprendizajes y utilizar en forma más activa los apoyos académicos, además de no centrar toda la mediación solamente en la unidad didáctica modular, integrar medios asincrónicos de comunicación para la tutoría y apoyo docente, mejorar la orientación académica sobre los elementos significativos de su aprendizaje, establecer estrategias que permitan al estudiante de primer ingreso matricular la asignatura como prioritaria y realizar un proceso de acompañamiento con otras asignaturas del programa de Humanidades.

Palabras clave: Educación a distancia, rendimiento escolar, objetivo educacional, Universidad Estatal a Distancia (UNED, Costa Rica).

\footnotetext{
* Doctor en Educación de la Universidad Estatal a Distancia (UNED), de Costa Rica. Magister en Bibliotecología con énfasis en gestión de información de la Universidad de Costa Rica, de Costa Rica. Licenciado en Administración Educativa de la UNED. Bachiller en Documentación y Bibliotecología de la Universidad Nacional, de Costa Rica. Profesor de Jornada Especial, en la UNED. Correo: jmezap@uned.ac.cr
} 


\title{
Elements that come into play in the process of academic adaptation according to the perception of a group of first-year students from UNED in the course on Methods of Distance Studies and Investigation
}

\begin{abstract}
In this article, the adaptation phenomenon of first-year students to the UNED, who were enrolled in Methods of Distance Studies and Investigation (code 00055) in the second quarter of 2019, is studied. This study is based on the analysis of the following categories: a) Categorization of the student in relationship to the integration of the principles of distance education including self-regulation, self-knowledge and academic motivation, b) Categorization of the students' perception in the teaching/learning process carried out in the subject matter $00055, \mathrm{c}$ ) Identification of the entities to which students had to reach out within the university to accompany their teaching/learning process, and d) Reception within the distance educational system. It is concluded that there is a need to improve the mediation process in the learning experiences and to more actively use academic support. This includes the need to not center all of the mediation only on a modular didactic unit, but also to integrate asynchronous means of communication for the tutoring session as well as educational support. There is also the need to improve the academic guidelines in the significant elements of the learning experience, by establishing strategies that allow each first-time student to register this course as a priority and undergo a process of accompaniment with other courses in the Humanities program.
\end{abstract}

Key words: Distance education, school performance, educational objective, National Distance University (UNED, Costa Rica).

\section{Éléments ayant une incidence dans le processus d'adaptation académique selon la perception d'un groupe d'étudiants de première année de l'UNED du cours Méthodes d'études à distance et de recherche}

\section{Résumé}

Cet article étude le phénomène de l'adaptation à l'UNED des étudiants de première année qui ont suivi le cours Méthodes d'études à distance et de recherche (code 00055) dans le deuxième quadrimestre du 2019. L'analyse part des suivantes catégories: a) Catégorisation de l'étudiant en rapport avec l'assimilation des principes de l'enseignement à distance (l'autorégulation, l'autoformation et la motivation académique), b) Catégorisation de la perception des étudiants du processus de l'enseignement apprentissage mené dans le cours 0055, c) Identification des instances auxquelles les étudiants ont recouru pour être appuyés dans son processus d'enseignement-apprentissage et d) Accueil dans la modalité d'enseignement à distance. Les résultats ont permis de conclure qu'il est nécessaire d'améliorer le processus de médiation des apprentissages et d'utiliser de manière plus active les soutiens académiques. En outre, de ne pas centrer toute la médiation dans le matériel didactique et d'intégrer des ressources asynchrones de communication pour le tutorat ainsi que pour le soutien pédagogique. D'ailleurs, il est important d'améliorer l'orientation académique chez les apprenants sur les éléments significatifs de leur apprentissage, ainsi que d'établir des stratégies pour que les étudiants puissent donner au cours un statut prioritaire dans leur formation; et de mettre en place un plan d'accompagnement dans les autres cours du programme des Humanités.

Mots-clés: Enseignement à distance, objectif d'apprentissage, réussite scolaire, Université de l'État à Distance (UNED). 


\section{Introducción}

La presente contribución es parte de la comunicación de los resultados de la investigación realizada en la Escuela de Ciencias Sociales y Humanidades (ECSH) sobre la percepción de un grupo de estudiantes sobre la asignatura Métodos de Estudios a Distancia e Investigación (código 00055) para indagar cuáles son los elementos centrales que inciden en el proceso de adaptación a la Universidad Estatal a Distancia (UNED).

El modelo y la asignatura se concentran en fomentar la incorporación de hábitos de estudio en los estudiantes, así como competencias y habilidades individuales para facilitar su autorregulación, el autoaprendizaje y la autoevaluación, que puede incidir en forma directa en el éxito académico y en la permanencia en la universidad. Elementos que forman parte del objeto epistémico de reflexión de la cátedra de Métodos de Estudios a Distancia e Investigación del Programa de Humanidades de la ECSH y son sujetos centrales de análisis los elementos que inciden en su adaptación a la modalidad a distancia de la educación superior.

Adicionalmente, en los últimos años se han realizado una serie de aportes que analizan la problemática de permanencia, seguimiento y asimilación del modelo de la UNED que conforman el estado de la cuestión y se constituye con los trabajos realizados por: Sofía Arburola Briceño ${ }^{1}$; Francia Alfaro Calvo y Brian Carranza Rodríguez ${ }^{2 ;}$ Leonardo Picado Rojas ${ }^{3}$; Luis Figueroa Retana $^{4}$; y Greivin Villegas Barahona ${ }^{5}$ quienes recogen evidencias del comportamiento, el esfuerzo que significa y la disciplina requerida para adaptarse a la dinámica y a las características del sistema de aprendizaje a distancia.

Adicionalmente, el trabajo realizado por Villegas ${ }^{6}$ identificó un grupo de variables predictoras del rendimiento académico en los estudiantes de la UNED, datos relevantes en el proceso de acompañamiento y formación de los hábitos de estudio individuales.

\footnotetext{
${ }^{1}$ Sofía Alburola Briceño, «Análisis del comportamiento de la matrícula regular ordinaria y de primer ingreso considerando los factores internos y externos que afectan a la Universidad Estatal a Distancia. Estudio basado en el periodo comprendido del año 2000 al 2015. Acuerdo tomado por el Consejo Universitario en sesión No. 1893-2016», San José: CIEI, 2016.

${ }^{2}$ Francia Alfaro Calvo y Bryan Carranza Rodríguez, «Informe Parcial de Perfil de Estudiantes de Primer Ingreso. Cohorte del I Cuatrimestre 2015», San José: CIEI, 2016.

${ }^{3}$ Leonardo Picado Rojas, "Análisis de la cohorte I-2015 de estudiantes de Primer ingreso a la Universidad Estatal a Distancia al III Cuatrimestre de 2016», San José: CIEI, 2017.

${ }^{4}$ Luis Fernando Figueroa Retana, «Causas de inactividad de los estudiantes de primer ingreso, en los Centros Universitarios de Alajuela, San Carlos, Desamparados y San Vito. Cohorte 1-2017» San José: CIEI, 2018.

${ }^{5}$ Grevin Villegas Barahona, «Modelo estadístico pedagógico para la toma de decisions administrativas y académicas con impacto en el mejoramiento continuo del rendimiento de los estudiantes universitarios, basado en los métodos de selección CUR» (tesis doctoral, Universidad de Salamanca, 2018).

${ }^{6}$ Ibíd.
} 
El proceso de adaptación de estudiantes al modelo de la UNED resulta complejo e inevitablemente requiere de esfuerzo para comprender el fenómeno y los elementos que inciden desde la propia percepción de sus actores, los contenidos que se brindan para formar a los estudiantes y las metodologías o estrategias que se deben desarrollar para lograr una adaptación al sistema en forma paulatina y efectiva.

La asignatura 00055 fue pensada como la puerta de entrada para la adquisición de esas habilidades para su integración y facilitar el proceso de adaptación de los estudiantes a la educación a distancia.

Además, se requiere complementar parte de los estudios realizados con la integración de la percepción de los estudiantes para contestar la siguiente pregunta de investigación: ¿Cuáles son los elementos cualitativos que influyen en la adaptación al modelo de educación a distancia de los estudiantes que ingresan a la UNED cada primer cuatrimestre?

El estudio es de tipo fenomenológico-hermenéutico; al respecto, José Tadeo Morales Carrillo ${ }^{7}$ señala que es relevante para reconocer los elementos de percepción subjetivas, pero facilita la construcción de un referente significativo para reconocer las bases teóricas del fenómeno.

La técnica de análisis de datos es el Muestreo Comparativo Constante (MCC) que, según Ana Cecilia Salgado Lévano ${ }^{8}$, permite confrontar las manifestaciones subjetivas con los resultados de las investigaciones reseñadas, o bien entre las opiniones de los entrevistados para indagar las categorías emergentes internas y externas que influyen desde la percepción de los estudiantes sobre el proceso de adaptación a la universidad y la posibilidad de evaluar el nivel de incidencia sobre esos factores.

Las categorías de análisis se establecieron con base en los elementos significativos identificados sobre la problemática de adaptación al modelo de la UNED, consignados en los documentos del estado de la cuestión, estableciando los siguientes enunciados con la finalidad de contestar la pregunta de investigación formulada:

-Categorización del estudiante en relación con los atributos e integración de los principios de la educación a distancia de autorregulación, autoconocimiento y motivación académica con base en la experiencia desarrollada en el II cuatrimestre del 2019.

-Categorización de la percepción de los estudiantes en el proceso de enseñanza aprendizaje realizado en la asignatura 0055. Pregunta por contestar: ¿Cuál es su percepción general de la asignatura de Métodos de

\footnotetext{
7 José Tadeo Morales Carrillo, «Fenomenología y Hermenéutica como Epistemología de la Investigación», Paradígma 32, n. ${ }^{\circ}$ 2, 2019: 007-022.

${ }^{8}$ Ana Cecilia Salgado Lévano, «Investigación cualitativa: diseños, evaluación del rigor metodológico y retos», Liberabit, Revista de Psicología 13, 2007: 71-78.
} 
Educación a Distancia sobre la adquisición de habilidades para el sistema de educación a distancia?

-Identificar las instancias que participaron y el papel que desarrollaron este cuatrimestre para acompañar su proceso de enseñanza aprendizaje ¿En quién se apoyó este cuatrimestre para estudiar en la UNED? ¿Qué trámites tuvo que realizar este cuatrimestre para atender sus necesidades de aprendizaje en la UNED? ¿A cuál instancia o dependencia tuvo que acudir? ¿Cómo calificaría la atención recibida para resolver su consulta en esa instancia?

-Categorización de la acogida en el sistema de educación a distancia con la pregunta: ¿Cómo valora su adaptación a la UNED? ¿Qué le gustaría que mejorara?

La selección de la población para ser entrevistada fue conformada por un grupo de estudiantes que matricularon la asignatura 00055 en el II cuatrimestre del 2019. La escogencia se realizó por conveniencia; al respecto, Roberto Hernández Sampieri, Carlos Fernández Collado y María del Pilar Baptista Lucio ${ }^{9}$ señalan que no requiere un muestreo estadístico y se acogió el interés de la cátedra de reunir testimonios de la percepción de los estudiantes de las zonas fuera de la capital sobre el proceso de acompañamiento que reciben y reunir su opinión sobre la asignatura, acogiendo la premisa empírica, que es el grupo poblacional del cual se conocen menos sus necesidades para adaptarse al sistema.

\section{Desarrollo}

El trabajo de campo se hizo por medio de llamadas telefónicas y fue dirigido a estudiantes oficialmente matriculados en la asignatura 00055, a quienes fue posible localizar y se manifestaron dispuestos a participar en el estudio. Se aclara que el número de personas participantes fue el necesario para alcanzar la saturación teórica que permitiera analizar el fenómeno. Es decir, la muestra no fue elaborada en función de su representación estadística y se finalizó la aplicación del instrumento al comprobar que no se aportaban nuevos elementos para la discusión en relación con los referentes teóricos consignados en el estado de la cuestión y en relación con los aportes individuales.

El total de entrevistados fue de 14 personas de las zonas Norte y Sur, Caribe, Cartago y Acosta (por el interés de la cátedra se excluyó la capital, pero se recomienda a futuro conformarla como una población de estudio del fenómeno).

\footnotetext{
${ }^{9}$ Roberto Hernández Sampieri, Carlos Fernández Collado y María del Pilar Baptista Lucio, Metodología de la Investigación, Bogotá: McGraw Hill, 2006.
} 
La identidad de los informantes y las entrevistas fueron realizadas en todos los casos con la aplicación ética, moral y consentimiento informado. El procesamiento de los audios se realizó con el programa Atlas TI para identificar los elementos de codificación y se utilizó como recurso para la protección de datos sensibles las notas del investigador para sistematizar las manifestaciones y los hallazgos expresados por los informantes.

Las características de la población entrevistada corresponden a las identificadas en los estudios institucionales realizados. Infiriendo que forman parte del promedio que cuatrimestralmente ingresa a la UNED y que se ubica en el rango etario entre 17 y 36 años; en promedio matriculan dos asignaturas, además existió paridad de sexo en el grupo de participantes.

\section{Categorización del estudiante en relación e integración de los principios de la educación a distancia de autorregulación, autoconocimiento y motivación académica}

El instrumento de indagación utilizó 18 elementos predictivos del rendimiento académico estudiados por Villegas ${ }^{10} \mathrm{y}$, recursivamente para el abordaje de la problemática, se agruparon en los principios de autorregulación, autoconocimiento y motivación.

Posterior a las entrevistas y al análisis de los datos recolectados, se estructuraron dos subcategorías emergentes como producto del análisis axial que las reagrupa en elementos internos correspondientes a las características individuales de los estudiantes, así como a los externos que son atribuibles a la influencia del modelo de la educación a distancia.

Se reconoce que las habilidades requeridas son la contenidas en el postulado del Modelo Pedagógico (MP) de la UNED ${ }^{11}$ :

-El sujeto aprendiz es un ente activo que elabora, de manera permanente, representaciones y procesos internos como resultado de su relación con el entorno físico y social; por lo tanto, interpreta y reinterpreta la realidad continuamente y en forma dinámica, a partir de sus estructuras cognitivas.

-El sujeto aprendiz tiende, en forma natural, a su autorregulación, lo cual lo conduce continuamente a autodeterminarse, autorrealizarse y trascender.

-Asimismo, es un ser en contexto humano que vive en relación con otras personas, pero tiende hacia la libertad y la conciencia para tomar sus propias decisiones. Por lo tanto, es un ente activo y constructor de su propia vida.

${ }^{10}$ Ibíd.

${ }^{11}$ UNED, «Modelo pedagógico», San José: UNED, 2004.

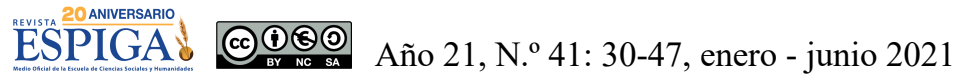


-El conocimiento tiene su origen en la interacción dialéctica entre el sujeto cognoscente y el objeto, dentro de un marco histórico-contextual del que forma parte el sujeto ${ }^{12}$.

Las personas entrevistadas mostraron evidencia de actuar con base en estas competencias, pero sobresale que varían en estrategias de distribución del tiempo, estilos de aprendizaje y en resultados académicos, como se rescata en las siguientes notas del investigador:

P3. Manifiesta que debe hacer una distribución de tiempo estratégica para atender sus obligaciones y estudiar, pues sale del trabajo a distintas horas.

P2. Manifiesta que su estilo es lento y lo califica de teórico tipo memorístico.

P12. Manifiesta reconocer que tiene problemas para mantener la atención y motivación para lograr la concentración y que fácilmente abandona la actividad cuando realiza la lectura, especialmente si agrega información complementaria a los temas centrales. Se le hace larga y aburrida. Lo confunde y desorienta.

Sobre los elementos de motivación académica al concluir el cuatrimestre, los entrevistados manifestaron estar satisfechos por el resultado académico. Se percibe una aceptación con resignación porque mencionaron que desearían tener un mejor rendimiento, pero fue limitado por las condiciones laborales y la dedicación del tiempo necesario para las asignaturas, como se consigna en las siguientes notas del investigador:

P3. Manifiesta que «ahí va». En relación con que logra salir con la actividad académica.

P2. La estudiante manifiesta que no es tan fácil, que le cuesta, a pesar de que se organiza y busca información adicional al libro. Manifiesta tener una actitud positiva. Pero, aunque lleva un control de estudio, no lo lleva exacto por la distribución de actividades cotidianas y laborales. Estudia por objetivos de aprendizaje. Manifiesta que no utiliza todos los recursos que le facilita la universidad.

También se logró consignar un caso donde se expresa no haber logrado compatibilizar las actividades personales, laborales y de estudio; esta persona abandonó la asignatura y la universidad al concluir el cuatrimestre.

12 Ibíd. 
Asimismo, en el proceso de análisis se identificaron elementos retomados de la propuesta de Villegas ${ }^{13}$, que son valorados de competencia directa del modelo de enseñanza de la UNED y que se posee la capacidad de planificar para cada asignatura.

Sobre estos elementos surge la pregunta epistémica: ¿Qué está sucediendo en este caso?

El primer elemento para reflexionar es el clima en clase o la tutoría, que es la práctica educativa que diferencia el modelo de educación a distancia del presencial, que posee el elemento del diálogo comunicativo sincrónico y las siguientes características:

Tutoría: es un recurso didáctico (formativo, pedagógico), de carácter voluntario, y tiene como propósito facilitar los procesos de aprendizaje del estudiante. Se realiza mediante una planificación sistemática, bidireccional, sincrónica o asincrónica y es llevada a cabo utilizando diferentes medios de comunicación. Tiene funciones académicas, de orientación, técnicas y administrativas $^{14}$.

En el proceso de entrevista se reunió evidencia de que las tutorías no se encuentran disponibles en igual número en el territorio nacional ni para todas las asignaturas, por lo que es habitual que los estudiantes deben acudir a otros centros universitarios; sin embargo, la situación de la alerta sanitaria del 2020 se manifiesta un cambio en la presencialidad del modelo y algunos no tan cercanos, como el caso de la estudiante de Upala, consignado en las notas del investigador:

P2. Manifiesta que las tutorías son buenas, pero no todas se dan en el CeU. Tuvo dos asignaturas con tutorías solamente en San José y no asistió porque no puede trasladarse por su trabajo y la distancia, además de residir en Upala. Lo resolvió por medio de WhatsApp y con apuntes de otros estudiantes.

Esta afirmación revela una condición compleja que efectivamente requiere atención en función del proceso de mediación de los aprendizajes, aunque la asistencia a estas no es de carácter obligatorio, es una aspiración que su disposición y difusión deben ser mayores o bien se deben utilizar los recursos tecnológicos para cubrir el territorio nacional, situación manifestada en la alerta sanitaria de la enfermedad COVID-19 del 2020.

13 Ibíd.

${ }^{14}$ UNED, Consecutivo Vicerrectoría Académica VA-03-682 del 19 junio de 2003. 
Igualmente, se logra reunir una percepción manifestada por estudiantes sobre la finalidad de la tutoría; existe la aspiración de que debe orientar claramente sobre los contenidos por evaluar y los instrumentos por entregar.

Surge la reflexión de si tanto los estudiantes y el modelo refuerzan la contradicción dicotómica entre una visión fragmentada de la tutoría como obligada a desarrollar los contenidos en función de la aprobación de las asignaturas y no en función de un proceso de formación de profesionales que requiere la sociedad costarricense para el bien común.

La utilidad de la tutoría también puede ser evidenciada en la asistencia de estas, pues las personas que acudieron reportaron un bajo número de asistencia después de la primera $\mathrm{y}$, los que no asistieron, manifestaron problemas de horario, o bien, que pudo prepararse sin ella para los exámenes.

Desde el punto de vista evaluativo, los entrevistados manifestaron adaptarse bien a las pruebas escritas, pero algunas personas mencionan que les fue difícil determinar lo relevante e importante para prepararse para los exámenes; y la tutoría, tampoco les permitió orientarse adecuadamente.

\section{Categorización de la percepción de estudiantes en el proceso de enseñanza aprendizaje realizado en la asignatura 00055}

El análisis de esta categoría se enmarca en la percepción de los estudiantes en las últimas dos semanas antes de terminar el cuatrimestre; por lo tanto, especialmente los resultados, metodológicamente, solamente pueden hacer inferencia a ese periodo. Además, según José Solano ${ }^{15}$, a la luz de la teoría de Vigostky, se evidencia un acercamiento a la zona próxima de aprendizaje, por consiguiente, es necesario un nuevo contexto educativo para visualizar el conocimiento alcanzado en la asignatura. En las notas del investigador se rescatan las siguientes manifestaciones de la percepción positivas de la asignatura:

P3. Está llevando esta asignatura después de dos cuatrimestres de ingresado a la universidad y manifiesta que el proceso de adaptación a la UNED probablemente se hubiera facilitado.

P5. Le sirvió para entender el sistema, las tutorías, formato de exámenes y la evaluación.

P14. Afirma que la asignatura le fue provechosa e importante, que le hubiera gustado llevarla antes pero no la pudo matricular por falta de cupo en el primer ingreso a la UNED.

P10. Le ayudó, pero la considera aburrida.

${ }^{15}$ José Solano Alpízar, «Educación y aprendizaje», San José: CECC-SICA, 2002.

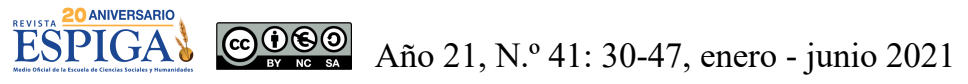


Un elemento de valoración que surge como atributo en el proceso de codificación axial es preguntarse sobre cuál es el rol de la asignatura. Inicialmente, en el imaginario de la UNED existe la aspiración que la asignatura 00055 sea la primera asignatura que cursen el estudiante al ingresar a la universidad. Sin embargo, en el estudio realizado por Leonado Picado ${ }^{16}$ se evidencia que no es una condición que se alcanza en la mayoría de los casos, no es necesariamente cierto y es una percepción institucional equivocada, pues es la población que matricula al final de periodo $\mathrm{y}$ mantienen prioridad los estudiantes antiguos en los cuatrimestres anteriores; según aproximaciones a los datos de cobertura de la población de primer ingreso para el segundo cuatrimestre del 2019, de 3300 de estudiantes de primer ingreso, el 18,19 \% no la cursaron, pese a que se realizó una autorización extraordinaria para la matrícula de ese periodo; por lo contrario, el porcentaje de personas que no la matriculan como primera asignatura de la vida universitaria sería mayor.

Adicionalmente, los estudiantes regularmente matriculan otra asignatura en forma conjunta, que fue planificada bajo el supuesto institucional de que el estudiante cursó o aprobó la asignatura 00055 conoce el modelo, se encuentra preparado, asimiló los principios básicos, además de que posee los hábitos y competencias del modelo de educación a distancia.

También, aparte de formar en los principios del modelo, el cometido de la asignatura es realizar una inducción a las principales dependencias de la universidad, pero se recolectó evidencia de que la primera reacción es reconocer los servicios relacionados a la matrícula y la beca; pero, llama la atención el poco reconocimiento de las instancias que brindan los servicios de apoyo académico como la biblioteca, audiovisuales y videoconferencia.

Sobre el material didáctico, se puede afirmar que es variado; sin embargo, en el proceso de entrevista surge la afirmación de que a los estudiantes les fue difícil reconocer en las unidades didácticas modulares lo relevante e importante y otras afirmaciones se dirigieron a identificar la extensión abundante de texto y los apoyos académicos.

\section{Indagación de las instancias que participaron y el papel desarrollado este cuatrimestre para acompañar su proceso de enseñanza aprendizaje}

En el proceso de indagación, y recursivamente para realizar el análisis, se reagruparon los elementos cualitativos manifestados por las personas entrevistadas en dos subcategorías emergentes: la primera, conformada por el papel que juegan las instancias formales u oficiales; y la segunda, por las

${ }^{16}$ Leonardo Picado Rojas, «Análisis de la cohorte..., 2017.

ESPIGA] CC) (1) (2) Año 21, N. ${ }^{\circ}$ 41: 30-47, enero - junio 2021 
instancias informales de acompañamiento que se constituyen por las acciones desarrolladas entre pares y se escapan al ámbito institucional.

Se logró confirmar las evidencias aportadas en los estudios institucionales reseñados, afirmando que el acompañamiento institucional es brindado por los servicios académicos ofrecidos en las sedes universitarias, las cátedras y profesores tutores, principalmente. Además, los más mencionados son matrícula, solicitud de beca, asistencia a tutorías, entrega de tareas, realización de pruebas y recoger resultados.

Reflexivamente, se debe valorar cada tutoría y servicio prestado a las personas estudiantes, así como su aporte al proceso de reforzamiento y asimilación de los contenidos. También, se debe ponderar las características comunicativas que juega la segunda subcategoría emergente, funcionalidad y la relevancia de la figura de los apoyos no institucionales o informales entre pares, particularmente, para que las tutorías sean un instrumento de mediación efectiva.

El acompañamiento informal o no oficial se caracteriza por ser una comunicación de «boca a boca» que se sobrepone a la comunicación y a los servicios institucionales. Además, posee los elementos cualitativos de ser ágil, pronta, basada en una experiencia previa (personal, casuística o referencial), es oportuna en algunos casos, puede ser individual o colectiva, es creíble y puede ser válida para tomar decisiones.

Adicionalmente, estos apoyos se configuran en elementos de influencia colectiva y se debe valorar significativamente por sus alcances, tal como mencionan Alfaro Calvo y Carranza Rodríguez ${ }^{17}$ : «Es igual de importante para jóvenes que para adultos».

También, se integra el elemento tecnológico, en particular las redes sociales, la comunicación de mensajería instantánea, interactividad tecnológica e interacción social. Solamente en la red social Facebook se identifican, según José Pablo Meza Pérez y Adriana Cascante Gatgens ${ }^{18}$, 284 perfiles relacionados a la UNED, donde 209 corresponden a dependencias y 72 a grupos autónomos que logra un alcance aproximado de 526481 seguidores. Algunos perfiles, como Estudiantes UNED, posee 21407 seguidores entre estudiantes, docentes y dependencias. Lo anterior representa un medio para la difusión de mensajes que abarca a una gran audiencia potencial.

Adicionalmente, en las entrevistas se recabó el dato de que los perfiles de redes sociales funcionan como centro de contactos para estudiantes, así como reclutamiento para conformar grupos de WhatsApp, pero no se logró conocer la cantidad de personas que utilizan este sistema de mensajería. Se puede inferir que muchos son efímeros y son integrados por un conjunto de

\footnotetext{
${ }^{17}$ Ibíd.

${ }^{18}$ José Pablo Meza Pérez y Adriana Cascante Gatgens, «Uso del Facebook asociados a la UNED», San José: CIEI, 2017.
} 
estudiantes matriculados en un cuatrimestre y para una asignatura en particular.

\section{Categorizar la acogida en el sistema de educación a distancia}

Se logró explorar cómo se desarrolla la acogida al sistema de educación a distancia; las personas entrevistadas identificaron que, inicialmente, en la UNED se realizó por medio de los centros universitarios y mediante las charlas de la Oficina de Orientación y Desarrollo Estudiantil de la Dirección de Asuntos Estudiantiles (DAES) después del proceso de matrícula.

En el proceso de entrevista se realizó la pregunta directa ¿Cómo valoraría su adaptación a la UNED? Que en términos de codificación en vivo, se rescata el atributo de que se califica como buena. Los estudiantes manifestaron reconocer que tienen varias de las habilidades de autorregulación y autoaprendizaje.

Se reunió evidencia de que en la valoración del modelo de adaptación a la UNED, los estudiantes lo relacionan en forma directa a su rendimiento académico y manifiestan su adaptación en función de la aprobación de todas las asignaturas del cuatrimestre.

En ninguno de los casos se logró reunir todas las características predictoras del modelo de Villegas ${ }^{19}$, pero es un indicativo que favorece la adquisición de algunas para la permanencia y que es necesario realizar otras para la adquisición de las características señaladas por el autor.

El factor de rendimiento académico es un elemento importante ligado a la percepción sobre la acogida, que es valorado como central y determinante. La adaptación y no adaptación es calificada fuertemente por parte del estudiantado en función de la aprobación de asignaturas o no.

\section{Conclusiones}

A manera de conclusión, se realiza un abordaje desde una reflexión sobre los atributos, propiedades y subcategorías para contestar la pregunta de investigación con base en las cuatro categorías de análisis.

a. Categorización del estudiante en relación e integración con los principios de la educación a distancia de autorregulación, autoconocimiento y motivación académica

19 Ibíd. 
La autorregulación, autoconocimiento y motivación académica requieren procesos que desarrollen directamente el estímulo de las funciones ejecutivas del cerebro en tres dimensiones esenciales, mencionadas por Ernesto Barceló Martínez, Soraya Lewis Harb y Mayilín Moreno Torres ${ }^{20}$ como la memoria de trabajo que permite al estudiante organizar la información, recordar a corto plazo y establecer las secuencias en los pasos lógicos para alcanzar la tarea y el control inhibitorio que permite al estudiante regular el estímulo automático, asociado a la motivación para no abandonar la actividad del estudio por la frustración de no comprender, por la falta de atención o distracciones del medio $\mathrm{y}$, por último, la dimensión de la flexibilidad cognitiva o mental que tiene relación con la capacidad de adaptarse, establecer prioridades y la capacidad de valorar otras perspectivas.

Además, según Melissa Aguzzi Fallas, Melissa Mora Umaña y Luis Ángel García Piedra $^{21}$, se reconoce el papel cognitivo de la memoria en los procesos formativos, el aprendizaje y el emocional como ejes de gran valor para la formación y apropiación del conocimiento que son inherentes al individuo.

La subcategoría externa es aquella en la cual la disponibilidad de la infraestructura, servicios, planeamiento y evaluación del proceso de enseñanza aprendizaje facilitan el reconocimiento del autoaprendizaje de estudiantes que teóricamente son abordados en la asignatura. Elementos que deben integrar la propuesta de acompañamiento a estudiantes y formar en estrategias para el uso de la memoria para el aprendizaje a distancia y no solamente en las asignaturas de la cátedra. La estrategia de formación y acompañamiento debe ser un contenido de todas las asignaturas rediseñadas del nuevo programa de Humanidades.

b. Categorización de la percepción de estudiantes en el proceso de enseñanza aprendizaje realizado en la asignatura 00055

En términos generales, la percepción de estudiantes es positiva y se valora como importante. También se reunió evidencia de que el material didáctico es abundante y requiere mucho tiempo abarcarlo en su totalidad, sumado a sus apoyos, lo cual puede limitar, efectivamente, la identificación y reconocimiento fácilmente, por parte de estudiantes, lo relevante e importante del material; especialmente si se valora que es una de las primeras experiencias en el modelo y muchos están en el proceso de formación de los principios de autorregulación y formando inicialmente el hábito de lectura.

\footnotetext{
${ }^{20}$ Ernesto Barceló Martínez, Soraya Lewis Harb y Mayilín Moreno Torres, «Funciones ejecutivas en Estudiantes universitarios que presentan bajo y alto rendimiento académico», Psicología desde el Caribe 18 (2006):109-138.

${ }^{21}$ Melissa Aguzzi Fallas, Melissa Mora Umaña y Luis Ángel García Piedra, «La memoria», San José: PROIFED, 2019.
} 
Se concluye que se debe valorar la cantidad de cupos de la asignatura, pues la dinámica de matrícula actual se prioriza a estudiantes antiguos $\mathrm{y}$, al finalizar, no son lo suficiente para acoger a toda la población de primer ingreso para acompañarla adecuadamente en el desarrollo de las habilidades requeridas en su adaptación a la universidad.

c. Identificar las instancias a las cuales debió acudir este cuatrimestre para acompañar su proceso de enseñanza aprendizaje

En términos generales, en las entrevistas realizadas, las personas logran enumerar pocas instancias, pero las menos reconocidas son los apoyos académicos como las tutorías, bibliotecas, audiovisuales, llamadas telefónicas, correos electrónicos y la radio, pero ninguna de las personas entrevistadas identificó que la utilizó.

Se concluye que hay una marcada dependencia por las unidades didácticas modulares escritas y se debe repensar el acompañamiento de las asignaturas con base en la pregunta: ¿Cuáles son los apoyos requeridos, su papel, relevancia para el acompañamiento y agregar un mayor valor a su utilización?

Adicionalmente, ante la pregunta epistémica ¿Qué está sucediendo en torno a este fenómeno?, surgió la categoría emergente «Recursos de información externos», que parte del atributo o la característica de que es una comunicación realizada de «boca a boca» entre pares.

Las redes sociales, formales e informales, surgen como una alternativa de acompañamiento por la inmediatez de la comunicación que supera a la institucional, pero son efímeras de corta vida, pues particularmente los grupos de WhatsApp se conforman en función de cada cuatrimestre y otros con estructuras más permanentes, como el grupo de Facebook Estudiantes UNED, que se ha convertido en un punto de referencia para acercar al estudiantado.

Otra forma de comunicación externa a la institucional es el acompañamiento cercano o individual entre estudiantes con atributos o características de ser de la misma comunidad, estar llevando la asignatura, haberla llevado o una afinidad familiar.

Se hace reflexión que en todos los casos de estudiantes que se mantuvieron en la universidad hasta el final de cuatrimestre, manifestaron de alguna forma tener un acompañamiento externo al institucional.

\section{d. Acogida en el sistema de educación a distancia}

Institucionalmente, se vislumbra necesario para la acogida de estudiantes al modelo de educación a distancia, el trabajo de la Oficina de Orientación y Desarrollo Estudiantil, como la instancia encargada de implementar el 
acompañamiento de estudiantes de la universidad, las sedes universitarias ${ }^{22}$ como la instalación física en los territorios, las áreas académicas para el establecimiento de la oferta de asignaturas, programas académicos, las cátedras de las asignaturas que se imparten y las instancias de apoyo académico como bibliotecas, recursos multimediales y tecnológicos.

Los apoyos familiares recibidos son un factor importante para la acogida y permanencia. Se identificó que las personas entrevistadas manifestaron el haber realizado ajustes a su dinámica hogareña que, en la mayoría de los casos, requirió de la reorganización de horarios para atender las obligaciones asignadas, redistribución del tiempo de las actividades de los integrantes y se evidenció que en algunos casos se cuenta con apoyos adicionales como familiares que asumen responsabilidades sobre el cuido de hijos, familiares, o bien, requirió de apoyos de otras personas que tienen conocimiento de los contenidos de las materias y conocen la gestión administrativa de la universidad, pues son o fueron estudiantes universitarios.

El factor laboral como elemento que interviene en la permanencia y adaptación del estudiante de la UNED es condicionante, pues en el caso de las personas trabajadoras, el cumplimiento del horario laboral siempre se impone incondicionalmente al tiempo que se debe asignar al estudio y de allí que manifiesten que deben ser responsables en función de cumplir con ambas actividades, pero relacionan su rendimiento académico como un reflejo en parte del tiempo real que pueden asignar al estudio.

Se concluye que eventualmente pueden existir otros trámites de servicios universitarios que son requeridos para el acompañamiento efectivo de los estudiantes en función de sus necesidades laborales y una mayor información para el uso de las orientaciones académicas.

Finalmente, un factor identificado como muy importante es la red de apoyos que mantienen estudiantes entre pares en el ámbito informal, estos reflejan un nivel muy importante para el apoyo en la contención de estudiantes en el proceso de adaptación al modelo de educación a distancia, en donde, al parecer, llenan los vacíos de la falta de orientación, información, motivación afectiva y acompañamiento.

Se concluye que la percepción de las personas entrevistadas es que institucionalmente son pocas las redes formales de apoyo institucional con actividades enfocadas en promover la permanencia. También, se evidenció que para llenar ese vacío se utilizan apoyos que se articulan en función de las redes sociales y grupos de mensajería como WhatsApp.

El proceso de adaptación en la universidad no finaliza en el primer cuatrimestre de ingreso, es una experiencia de vida estudiantil en la cual intervienen variables personales, condiciones sociales, educativas, económicas y otras en las cuales la universidad puede inferir, pero es una experiencia vivencial e individual de un proyecto de vida en construcción, no

${ }^{22}$ Ibíd. 
es un proceso lineal o fácil de intervenir pues existen múltiples actores que participan de forma directa e indirecta.

\section{Recomendaciones}

En términos de adaptación al sistema de educación a distancia, la propuesta de Villegas $^{23}$ logra identificar las principales variables predictoras del rendimiento académico y se puede inferir que, entre mayor sea la adquisición de esas variables, la posibilidad de éxito académico será mayor.

Se recomienda la integración de una red institucional de apoyo al proceso de acompañamiento con características formales, informales e híbridas. Inicialmente, centrarse en los principales elementos mencionados por las personas entrevistadas, pero dirigidos en orientar en los procesos de admisión a la universidad, características y competencias necesarias para el proceso de adaptación a la UNED, así como trámites para la escogencia de asignaturas a matricular, orientación sobre la distribución del tiempo y criterios de valoración para escoger la carga académica.

Adicionalmente, esta red de apoyo al proceso de acompañamiento, debe integrar a todas las dependencias involucradas formales e informales, inicialmente identificadas en esta investigación y otras que surjan.

Se recomienda priorizar el proceso de matrícula para favorecer a estudiantes de primer ingreso e influir para que logren cursar el bloque de Humanidades en los primeros cuatrimestres.

Atender, en forma prioritaria, la cobertura de tutorías a nivel nacional y favorecer mecanismos asincrónicos para facilitar la atención a la población que no puede asistir a ellas por motivos de distancia y horarios de trabajo.

Mejorar la comunicación de orientaciones académica para la mediación de los aprendizajes que faciliten al estudiante identificar claramente los contenidos puntuales y centrar la evaluación no en contenidos, sino en el desarrollo de evidencias del aprendizaje realizado por parte del estudiante.

En términos de contenidos, integrar el tema del papel de la educación superior para el desarrollo de profesionales que la sociedad costarricense requiere para el bien común, con la intensión de visualizar el valor público del sistema de educación superior. Además, integrar el desarrollo de las habilidades de la memoria en el proceso de aprendizaje en el modelo de la educación a distancia, reseñados por Aguzzi Fallas, Mora Umaña y García Piedra ${ }^{24}$. Adicionalmente, integrar estrategias de entrenamiento de las funciones ejecutivas del cerebro para la autorregulación del aprendizaje en todas las nuevas asignaturas del programa de Humanidades.

${ }^{23}$ Ibíd.

${ }^{24}$ Melissa Aguzzi Fallas, Melissa Mora Umaña y Luis Ángel García Piedra, «La memoria»... 2019.

ESPIGAd @) (1) (2) Año 21, N. o 41: 30-47, enero - junio 2021 


\section{Formato de citación según APA}

Meza-Pérez, J. (2021). Elementos que inciden en el proceso de adaptación académica según la percepción de un grupo de estudiantes de primer ingreso de la UNED de la asignatura Métodos de Estudio a Distancia e Investigación. Revista Espiga, 21 (41), páginas 30-47.

\section{Formato de citación según Chicago-Deusto}

Meza-Pérez, José Pablo. «Elementos que inciden en el proceso de adaptación académica según la percepción de un grupo de estudiantes de primer ingreso de la UNED de la asignatura Métodos de Estudio a Distancia e Investigación». Revista Espiga 21, n. ${ }^{\circ} 41$ (enero-junio, 2021): páginas 30-47.

\section{Referencias bibliográficas}

Aguzzi Fallas, Melissa, Melissa Mora Umaña y Luis Ángel García Piedra. «La memoria», San José: PROIFED, 2019.

Alburola Briceño, Sofía. «Análisis del comportamiento de la matrícula regular ordinaria y de primer ingreso considerando los factores internos y externos que afectan a la Universidad Estatal a Distancia. Estudio basado en el periodo comprendido del año 2000 al 2015. Acuerdo tomado por el Consejo Universitario en sesión No. 1893-2016». San José: CIEI, 2016.

Alfaro Calvo, Francia y Bryan Carranza Rodríguez. «Informe Parcial de Perfil de Estudiantes de Primer Ingreso. Cohorte del I Cuatrimestre 2015». San José: CIEI, 2016.

Barceló Martínez, Ernesto, Soraya Lewis Harb y Mayilín Moreno Torres. «Funciones ejecutivas en estudiantes universitarios que presentan bajo y alto rendimiento académico». Psicología desde el Caribe 18 (2006):109-138.

Figueroa Retana, Luis Fernando. «Causas de inactividad de los estudiantes de primer ingreso, en los Centros Universitarios de Alajuela, San Carlos, Desamparados y San Vito. Cohorte 1-2017». San José: CIEI, 2018.

Hernández Sampieri, Roberto, Carlos Fernández Collado y María del Pilar Baptista Lucio. Metodología de la Investigación. Bogotá: McGraw Hill, 2006.

Meza Pérez, José Pablo y Adriana Cascante Gatgens. «Uso del Facebook asociados a la UNED». San José: CIEI, 2017.

Morales Carrillo, José Tadeo. «Fenomenología y Hermenéutica como Epistemología de la Investigación». Paradígma 32, n. ${ }^{\circ} 2$ (2019): 007-022. 
Picado Rojas, Leonardo. «Análisis de la cohorte I-2015 de estudiantes de primer ingreso a la Universidad Estatal a Distancia al III Cuatrimestre de 2016». San José: CIEI, 2017.

Salgado Lévano, Ana Cecilia. «Investigación cualitativa: diseños, evaluación del rigor metodológico y retos». Liberabit, Revista de Psicología 13 (2007): 71-78.

Solano Alpízar, José. «Educación y aprendizaje». San José: CECC-SICA, 2002. UNED. «Modelo pedagógico». San José: UNED, 2004.

Villegas Barahona, Grevin. «Modelo estadístico pedagógico para la toma de decisiones administrativas y académicas con impacto en el mejoramiento continuo del rendimiento de los estudiantes universitarios, basado en los métodos de selección CUR» (tesis doctoral, Universidad de Salamanca, 2018). 